\title{
Cross-Sectional Analysis of the Associations Between Four Common Cancers and Disability
}

\author{
Lisa I. lezzoni, MD, MSc ${ }^{1,2}$; Sowmya R. Rao, $\mathrm{PhD}^{3,4}$; Nicole D. Agaronnik, $\mathrm{BS}^{1}$; and Areej El-Jawahri, $\mathrm{MD}^{2}$
}

\begin{abstract}
Background: Approximately 61 million Americans have a disability. Little research has explored whether disability is associated with subsequent diagnosis of cancer, the second-leading cause of death in the United States. The objective of this study was to explore associations between cancer and disability, focusing on 4 cancers that may present with nonspecific symptoms that could be conflated with aspects of disability, thus delaying cancer diagnoses. An analysis of a nationally representative survey using sampling weights to produce national estimates was performed. Methods: Civilian, noninstitutionalized US residents responding to the 2010-2017 National Health Interview Surveys totaling 259,392 Sample Adult Core survey respondents were included. We used self-reported functional status limitations to identify persons with movement difficulties (MD), complex activity limitations (CAL), and no disability. Multivariable regressions predicting cancer diagnosis included sociodemographic characteristics, tobacco use, body mass index, access to care indicators, and disability status. Results: Persons with preexisting disability had significantly higher rates of cancer (ranging from 0.40 [SE, 0.05] for ovarian to 3.38 [0.14] for prostate) than did those without disability $(0.20$ [0.02] and 1.26 [0.04] for the same cancers; all $P<.0001$ ). Multivariable analyses found strong associations of preexisting $\mathrm{MD}$ and $\mathrm{CAL}$ with colorectal cancer, with adjusted odds ratios (aORs) of $1.5(95 \% \mathrm{Cl}, 1.2-1.9)$ and $1.9(1.5-2.4)$, respectively. For non-Hodgkin's lymphoma, the aOR for CAL was 1.5 (1.1-2.1). For prostate cancer, aORs for MD were $1.2(1.0-1.3)$ and 1.1 (1.0-1.3) for CAL. Using cross-sectional survey data, we could only identify statistical associations, not causality. Conclusions: Our populationbased analyses suggest that persons with disability may constitute a high-risk population, with higher cancer incidence. Optimizing appropriate screening and fully investigating new signs and symptoms are therefore critical for patients with disability.
\end{abstract}

J Natl Compr Canc Netw 2020;18(8):1031-1044 doi: 10.6004/jnccn.2020.7551

${ }^{1}$ Health Policy Research Center-Mongan Institute, Massachusetts General Hospital; ${ }^{2}$ Department of Medicine, Harvard Medical School; ${ }^{3}$ Massachusetts General Hospital Biostatistics Center; and ${ }^{4}$ Boston University School of Public Health, Boston, Massachusetts.

\section{Background}

Approximately 61 million Americans, $26 \%$ of the US population, have some type of disability, and this population is increasing. ${ }^{1}$ According to 2017 National Health Interview Survey (NHIS) data, 8.5\% of Americans aged $\geq 18$ years have-or have had-cancer, ${ }^{2}$ the second leading cause of death in the United States. ${ }^{3}$ Although these disability and cancer prevalence estimates are independent, clinicians have recognized increasingly that cancer and its treatment can be profoundly disabling, often causing neurologic or musculoskeletal impairments requiring rehabilitation therapy. ${ }^{4-6}$ In 2017, $2.8 \%$ of beneficiaries receiving Social Security Disability Insurance qualified because of cancer-related disability. ${ }^{7}$ The Social Security Administration's Compassionate Allowances offers fast-track approval of disability benefits for person with progressive cancer. ${ }^{8}$

However, whether persons who already have disability are more likely than nondisabled persons to develop cancer has received little study. Several factors suggest that preexisting disability and cancer could be associated. Persons with disability have higher rates of tobacco use $^{9}$ and obesity, ${ }^{10}$ potential cancer risk factors. ${ }^{11,12}$ Persons with disability have higher rates of poverty, ${ }^{13,14}$ which is associated with increased cancer mortality risks. Additionally, persons with disability can experience disparities in cancer screening, ${ }^{15-17}$ which may result in failures to detect precancerous lesions. For older individuals with early-onset disability, prior patterns of care (eg, frequent radiographs and thus radiation exposure during childhood) could also increase cancer risks. Persons with disability, either congenital or acquired by midlife, show physiologic signs of more rapid aging compared with nondisabled persons, although reasons for this are unknown. ${ }^{18-20}$ Might this heighten risks of developing cancer?

One separate but related question is whether, when persons with disability do develop cancer, they receive timely diagnoses. Some groups that experience healthcare 
disparities also face delayed cancer diagnoses. ${ }^{21,22} \mathrm{Di}-$ agnostic overshadowing-when clinicians erroneously attribute cancer signs and symptoms to the patient's underlying disability—can potentially delay cancer diagnoses. ${ }^{23}$

This research explored associations between cancer and disability, focusing on 4 cancers that may present with nonspecific symptoms that could be conflated with aspects of disability. Few datasets contain information on both cancer and disability (eg, the SEER cancer registry does not report functional status information). We therefore analyzed data from the NHIS, a nationally representative survey of civilian, noninstitutionalized Americans, which asks about common health conditions, including cancer and functional status. Our major analytic goals were to examine (1) population prevalence among adults with 4 cancer diagnoses and disability, (2) associations between cancer diagnoses and preexisting disability, (3) age at cancer diagnosis for persons with versus without preexisting disability, and (4) associations between cancer diagnosis and preexisting disability after adjusting for sociodemographic characteristics and selected risk factors.

\section{Methods}

\section{Data}

We downloaded 2010-2017 NHIS Public Release data from the National Center for Health Statistics (NCHS) website. The NHIS Basic Module contains 3 questionnaires: Family Core, Sample Adult Core, and Sample Child Core. The Family Core collects information on all family members. One randomly selected person aged $\geq 18$ years within each family receives the Sample Adult Core questionnaire, which gathers more detailed health history and functional status information. If the randomly sampled adult is physically or cognitively unable to respond or is unavailable, a knowledgeable adult family member provides proxy responses. In 2017, for example, the NHIS interview sample included 32,617 households, and the Sample Adult Core included 26,742 individuals, 423 with proxy responses. The household response rate was $66.5 \%$; the conditional response rate for the Sample Adult Core was $80.7 \% .^{24}$

NHIS oversamples black, Hispanic, and Asian populations, and minorities aged $\geq 65$ years. NHIS's associated sampling weights, based on its complex sample design, support nationally representative estimates for civilian, noninstitutionalized populations. All analyses used these sampling weights.

\section{Chronic Disability Indicators}

To identify chronic disability, we began with NCHS algorithms capturing limitations in body functions and activity and participation difficulties. ${ }^{22}$ These algorithms use responses from the Adult Health Status and Limitations module in the Sample Adult Core questionnaire. Supplemental eAppendix 1 (available with this article at JNCCN.org) presents the question stems and description of response categories.

After NCHS's approach, we combined responses from various functional and activity limitation questions to create 2 broad categories of disability-basic action difficulties (BADs) and complex activity limitations (CALs) with 7 subcategories, which include movement, sensory, emotional, or cognitive difficulties, and self-care, social, or work limitations (see supplemental eAppendices 1 and 2). The 7 categories are not conceptually mutually exclusive: individual BADs can contribute to CALs.

To identify persons with chronic disability, we refined the NCHS algorithms. When participants indicate functional status difficulties, NHIS asks how long the difficulties have lasted. We considered difficulties present for at least a year to be chronic. We combined participants who reported nonchronic $(<1$ year duration) conditions with those without the particular disability.

\section{Identifying Cancer, Time Frames, and} Other Variables

Sample Adult Core respondents were asked "if they have ever been told by a doctor that they had cancer," and if so, the type of cancer. We considered 4 cancer types: colorectal cancer (CRC), non-Hodgkin's lymphoma (NHL), ovarian cancer, and prostate cancer. We also obtained age at diagnosis reported by participants and computed time since cancer diagnosis. We then compared durations for the reported disability and time since cancer diagnosis in order to determine whether disability was preexisting or occurred subsequent to the cancer. For respondents reporting $>1$ disability type, we used the earliest disability in comparing disability with time from cancer diagnosis. We categorized respondents into those with disability occurring before cancer, simultaneously with cancer, and after the cancer diagnosis.

We used Sample Adult Core questions to identify basic sociodemographic characteristics that could be important risk factors ${ }^{14}$ (age, race, ethnicity, education, health insurance status, usual source of care, body mass index $[\mathrm{BMI}]$, and smoking status), except for income, which came from Family Core responses linked to the Sample Adult participant. We grouped age into 4 categories ( $<45,45-64,65-79, \geq 80$ years). For prostate cancer analyses, we combined the 2 younger categories (age $<65$ ) because of small numbers in the age $<45$ group.

\section{Analysis}

We conducted all analyses using SAS 9.4 (SAS Institute Inc.) and SUDAAN 11.0.3 (RTI International). To account for NHIS's complex sampling design, we included weights, 
cluster, and strata variables in all analyses. We combined the data across years (2010-2017). We obtained summary statistics (proportions, means, SEs) for all variables. We used 2-sided chi-square tests to evaluate the association of having cancer with the different disability types. We focus our presentation here on 2 common disability types with an adequate sample size for rigorous analysis: movement difficulty (MD) and any CAL. In our analyses, persons with no disability had none of the 7 disability types.

We obtained odds ratios (ORs) and 95\% CIs from separate multivariable logistic regressions assessing the relationship of MD and CAL with each of the cancer diagnosis, adjusting for age $(<45,45-64,<65$ for prostate cancer, 65-79, and $\geq 80$ years), race (white, black, Asian, other/multiple races), Hispanic ethnicity (yes/no), education (less than high school, high school, some college/associate's degree, college/advanced degree), BMI ( $<25,25-29,30-34,35-39$, and $\left.\geq 40 \mathrm{~kg} / \mathrm{m}^{2}\right)$, smoking status (current, former, never), family income $<200 \%$ of the poverty threshold (yes, no), health insurance (covered, not covered), and usual source of care (yes, no). Models to assess the relationship of disability with CRC and NHL also included sex (male, female) in the models. All tests were 2-sided, and we considered $P$ values $<.05$ to be statistically significant.

\section{Results}

From 2010 to 2017, the NHIS Sample Adult Core involved 259,392 participants. Table 1 shows the distribution, overall and by sex, of the sociodemographic characteristics of these participants and risk factors (BMI and smoking status) and rates of each of the 4 cancers and different disability types. Across men and women, $0.6 \%$ (SE, $0.02 \%$ ) had ever been told they had CRC and $0.3 \%$ (SE, $0.01 \%$ ) reported NHL; among men, $2.2 \%$ (SE, $0.05 \%$ ) reported having been told they had prostate cancer; and $0.4 \%$ (SE, $0.02 \%$ ) of women reported ovarian cancer. MD was the most common disability type, reported by $20.9 \%$ (SE, $0.1 \%$ ) of men and women.

\section{Identifying Preexisting Disability}

Our major research questions focused on patients who had disability before their cancers. We concentrate here on 2 disability types: MD and any CAL. For each of the 4 cancer types, Figure 1 shows the percent of cases reporting the timing of their disability relative to their cancer diagnosis: before, simultaneously with, or after the cancer diagnosis. For CRC, prostate cancer, and NHL, $>60 \%$ of persons reported their MD or CAL occurred before their cancer; for ovarian cancer, approximately $50 \%$ reported preexisting MD or CAL. Results presented herein focus only on persons who had MD or CAL disability before being diagnosed with cancer.
Cancer Rates and Age at Cancer Diagnosis by Preexisting Disability Status

The top half of Table 2 shows the percent (SE) of participants reporting cancer by preexisting disability status (MD or CAL). Persons with preexisting disability had significantly higher rates of cancer (ranging from $0.40 \%$ [0.05\%] for ovarian to $3.38 \%$ [0.14\%] for prostate) than did those without disability $(0.20 \%[0.02 \%]$ and $1.26 \%[0.04 \%]$ for the same cancers, all $P<.0001)$. For example, among men who already had MD, $3.38 \%(0.14 \%)$ reported a prostate cancer diagnosis compared with $1.26 \%(0.04 \%)$ of men without disability.

The bottom half of Table 2 shows the mean age (SE) in years at cancer diagnosis for persons without disability versus those with preexisting MD or CAL. In all comparisons, persons with preexisting disability were significantly older (by 3-15 years) when diagnosed with cancer than those without disability (most $P<.0001$ ). For example, persons with preexisting MD were on average aged $62.6(0.8)$ years when diagnosed with CRC, compared with $55.9(0.7)$ years for persons without disability $(P<.0001)$.

\section{Accounting for Sociodemographic and Other Characteristics}

For each cancer, Table 3 shows bivariable associations for sociodemographic characteristics, risk factors, and time from cancer diagnosis for persons with and without preexisting MD or CAL disability. For all 4 cancers, compared with persons without disability, those with MD or CAL disability were older, had lower education levels (CAL disability only for ovarian cancer), and were more likely to have incomes $<200 \%$ of the federal poverty level. Health insurance status and having a usual source of care patterns vary by cancer: for CRC, NHL, and ovarian cancer, persons with disability are substantially more likely to have insurance coverage than those without disability. Compared with persons without disability, those with MD and CAL disability have substantially higher BMIs for CRC, prostate cancer, and ovarian cancer. Compared with persons without disability, persons with MD or CAL disability were substantially more likely to have positive smoking histories (current or former smoker) for all 4 cancers. Persons with MD or CAL disability had significantly shorter time from cancer diagnosis than individuals without disability for CRC and NHL.

We produced ORs and 95\% CIs from multivariable models assessing the relation of cancer diagnoses with preexisting disability status accounting for sociodemographic and risk factor characteristics. The question was whether accounting for these other characteristics would attenuate or eliminate the association of disability with cancer diagnosis. We performed 8 regressions: for each of the 4 cancers and for each of the 2 preexisting disability types, MD and CAL. 
Table 1. Distribution of Sociodemographic Characteristics, Disability, and Cancer by Sex ${ }^{a}$

\section{Variable}

Total, N

Sociodemographic characteristics

Mean age (SE), y

Age

$$
<45 \mathrm{y}
$$

45-64 y

$65-79$ y

$\geq 80 \mathrm{y}$

Race

White
Black
Asian
Other/Mixed

Hispanic ethnicity

Education

\begin{tabular}{ll}
\hline Less than high school & $13.7(0.2)$ \\
\hline High school & $26.6(0.2)$ \\
\hline Some college/associate's degree & $29.5(0.2)$ \\
\hline College/Advanced degree & $30.1(0.3)$
\end{tabular}

Body mass index

\begin{tabular}{lc}
\hline$<25 \mathrm{~kg} / \mathrm{m}^{2}$ & $29.7(0.2)$ \\
\hline $25-29 \mathrm{~kg} / \mathrm{m}^{2}$ & $40.9(0.2)$ \\
\hline $30-34 \mathrm{~kg} / \mathrm{m}^{2}$ & $19.2(0.2)$ \\
\hline $35-39 \mathrm{~kg} / \mathrm{m}^{2}$ & $6.8(0.1)$ \\
$\geq 40 \mathrm{~kg} / \mathrm{m}^{2}$ & $3.4(0.1)$
\end{tabular}

Smoking status

Current smoker
Former smoker
Never-smoker
Smoker, current status unknown

Family income below FPT

Has health insurance

Has a usual source of care

Cancer history

Ever told had colorectal cancer

Mean age at diagnosis, $y$

Ever told had non-Hodgkin's lymphoma

Mean age at diagnosis, $\mathrm{y}$

Ever told had prostate cancer

Mean age at diagnosis, $y$

Ever told had ovarian cancer

Mean age at diagnosis, y
Male
$\%$ (SE)

$46.1(0.1)$

$48.3(0.3)$

$34.7(0.2)$

$13.3(0.1)$

$3.7(0.1)$

$80.5(0.3)$

$11.3(0.2)$

$5.5(0.1)$

$2.7(0.1)$

$15.7(0.3)$

$13.7(0.2)$

$6(0.2)$

$30.1(0.3)$

$29.7(0.2)$

$0.9(0.2)$

$.2(0.2)$

$3.4(0.1)$

$19.1(0.2)$

$25.4(0.2)$

$55.5(0.2)$

$0.03(0.01)$

$11.7(0.2)$

$84.3(0.2)$

$81.3(0.2)$

$0.6(0.03)$

$57.9(0.6)$

$0.3(0.02)$

49.0 (1.2)

$2.2(0.05)$

$62.9(0.3)$
Female

$\%$ (SE)

143,326

$47.6(0.1)$

$46.9(0.1)$

$41.6(0.9)$

Total

$\%$ (SE)

259,392

$45.8(0.2)$

$47.0(0.2)$

$34.3(0.2)$

$34.5(0.2)$

$14.6(0.1)$

$14.0(0.1)$

$5.3(0.1)$

$4.5(0.1)$

78.5 (0.3)

$12.9(0.2)$

$79.5(0.2)$

$5.7(0.1)$

$12.2(0.2)$

$2.8(0.1)$

$5.6(0.1)$

$14.5(0.3)$

$2.8(0.1)$

$15.1(0.3)$

$12.9(0.2)$

$13.3(0.2)$

$24.9(0.2)$

$25.7(0.2)$

$32.1(0.2)$

$30.9(0.2)$

$30.1(0.3)$

$30.1(0.3)$

$42.7(0.2)$

$36.3(0.2)$

$28.3(0.2)$

$34.5(0.1)$

$16.2(0.1)$

$17.7(0.1)$

$7.2(0.1)$

$7.0(0.1)$

$5.6(0.1)$

$4.5(0.1)$

$14.9(0.2)$

$16.9(0.1)$

$18.9(0.2)$

$22.0(0.1)$

$66.3(0.2)$

$61.1(0.2)$

$0.03(0.01)$

$0.03(0.01)$

$15.2(0.2)$

$13.6(0.2)$

$87.7(0.2)$

$86.1(0.2)$

$89.4(0.1)$

$85.5(0.1)$

$0.5(0.02)$

$0.6(0.02)$

$58.3(0.7)$

$58.1(0.5)$

$0.2(0.02)$

$0.3(0.01)$

50.7 (1.3)

\section{$49.7(0.9)$}

$0.4(0.02)$




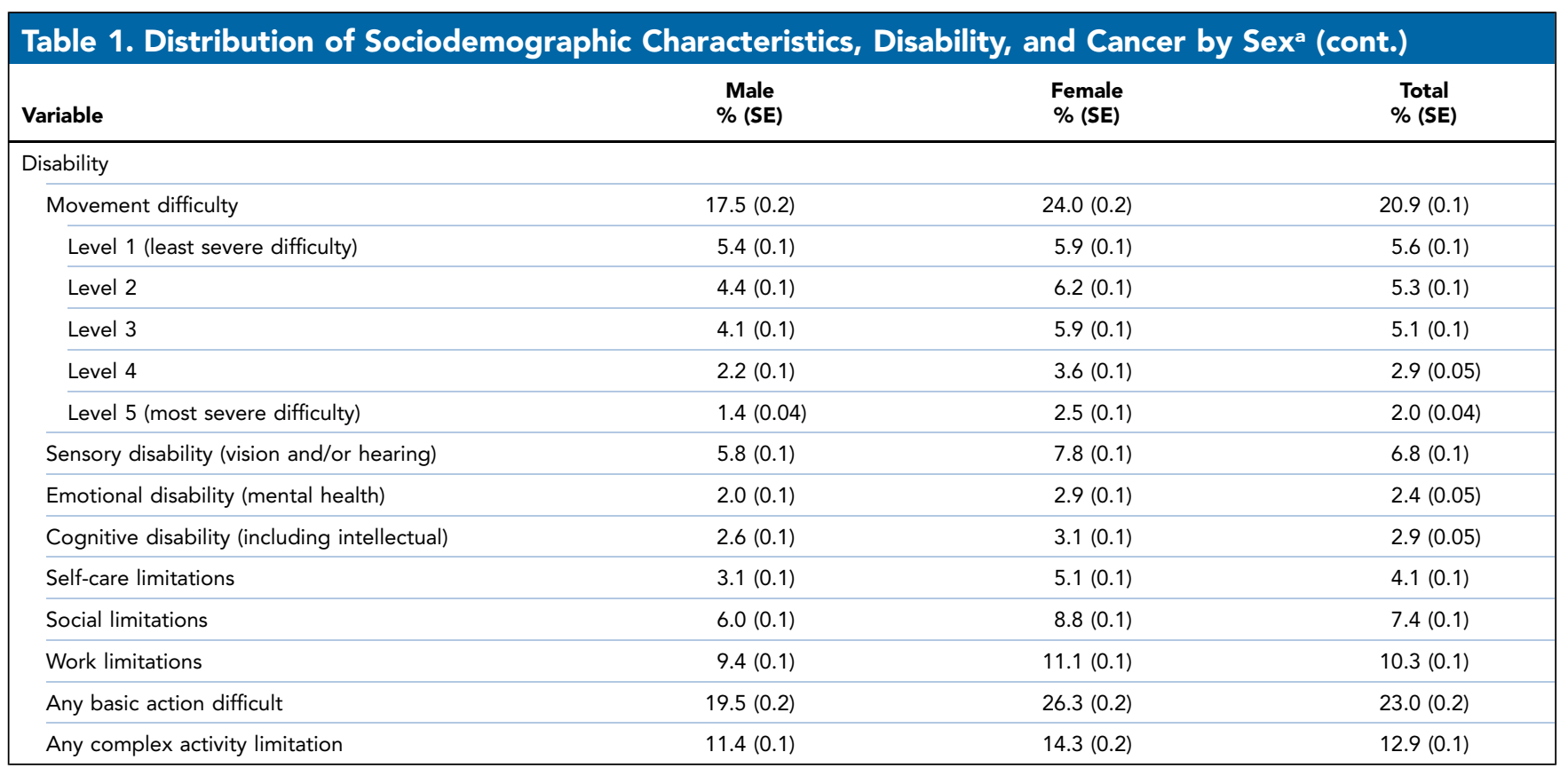

Abbreviation: FPT, federal poverty threshold.

${ }^{a}$ All analyses accounted for the complex sampling design and used National Health Interview Survey sampling weights to produce nationally representative estimates for civilian, noninstitutionalized US residents.

Table 4 shows results from these 8 multivariable regressions, presenting adjusted odds ratios (aORs) with 95\% CIs; reference groups have aORs of 1.0. Even after adjusting for other covariates, preexisting MD and CAL were strongly associated with CRC, with aORs (95\% CI) of 1.5 (1.2-1.9) and 1.9 (1.5-2.4), respectively. For NHL, the aORs for MD and CAL were $1.2(0.9-1.6)$ and 1.5 (1.1-2.1), respectively. In contrast, for prostate cancer, the aOR

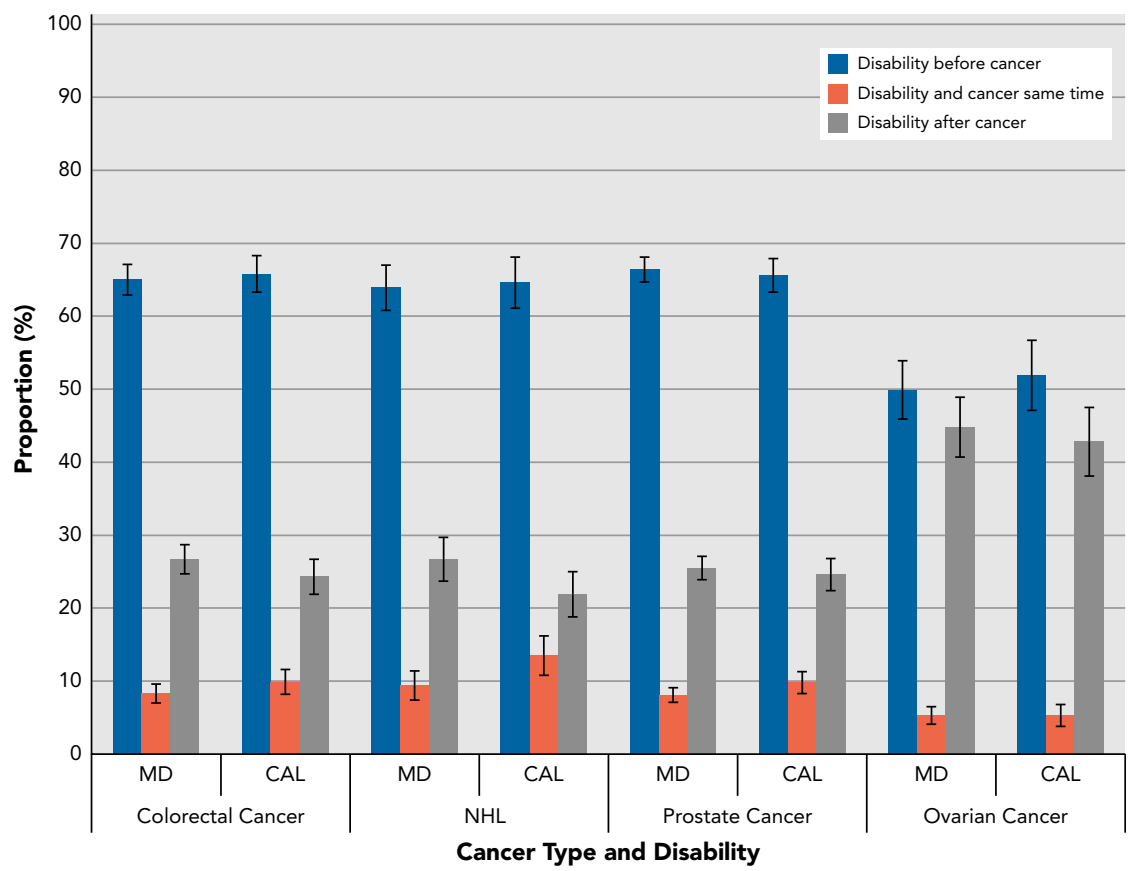

Figure 1. Time between disability and cancer.

Abbreviations: CAL, complex activity limitations; MD, movement difficulty; NHL, non-Hodgkin's lymphoma. 


\begin{tabular}{|c|c|c|c|c|c|}
\hline & No Disability & MD & $P$ Value $^{\mathrm{b}}$ & Any CAL & $P$ Value $^{b}$ \\
\hline Total, N & 183,106 & 61,291 & & 38,862 & \\
\hline \multicolumn{6}{|l|}{ Cancer rate, \% (SE) } \\
\hline Colorectal cancer & $0.24(0.01)$ & $0.85(0.05)$ & $<.0001$ & $1.02(0.07)$ & $<.0001$ \\
\hline Non-Hodgkin's lymphoma & $0.17(0.01)$ & $0.32(0.03)$ & $<.0001$ & $0.39(0.04)$ & $<.0001$ \\
\hline Prostate cancer & $1.26(0.04)$ & $3.38(0.14)$ & $<.0001$ & $3.13(0.18)$ & $<.0001$ \\
\hline Ovarian cancer & $0.20(0.02)$ & $0.40(0.05)$ & $<.0001$ & $0.47(0.07)$ & $<.0001$ \\
\hline \multicolumn{6}{|c|}{ Age at cancer diagnosis, mean (SE), y } \\
\hline Colorectal cancer & $55.9(0.7)$ & $62.6(0.8)$ & $<.0001$ & $61.3(0.9)$ & $<.0001$ \\
\hline Non-Hodgkin's lymphoma & $44.8(1.3)$ & $61.0(1.3)$ & $<.0001$ & $59.3(1.6)$ & $<.0001$ \\
\hline Prostate cancer & $62.2(0.4)$ & $66.0(0.5)$ & $<.0001$ & $64.9(0.7)$ & $<.001$ \\
\hline Ovarian cancer & $40.2(1.4)$ & $45.5(2.2)$ & .04 & $45.8(2.6)$ & .05 \\
\hline
\end{tabular}

Abbreviations: CAL, complex activity limitation; MD, movement difficulty; NHIS, National Health Interview Survey.

aAll analyses accounted for the complex sampling design and used NHIS sampling weights to produce nationally representative estimates for civilian, noninstitutionalized US residents.

${ }^{\mathrm{b}}$ Comparing persons with no disability versus those with MD or CAL disability.

for MD was 1.2 (1.0-1.3) and 1.1 (1.0-1.3) for CAL. For ovarian cancer, the aOR for MD was $1.4(0.9-2.1)$ and 1.5 (0.9-2.4) for CAL.

\section{Discussion}

Disability rates increase with aging, ${ }^{25}$ as do rates of cancer. ${ }^{26}$ Therefore, it was not surprising to find that persons with preexisting disability had significantly higher rates of cancer than those without disability, and that persons with preexisting disability generally had their cancers diagnosed at later ages than those without disability. Other factors often associated with cancer, such as obesity, ${ }^{12}$ tobacco use, ${ }^{11}$ and poverty, ${ }^{27}$ are also associated with disability, again suggesting possible links between preexisting disability and cancer. Notably, however, even after adjusting for these various confounding factors, we found associations between at least 1 preexisting disability type-MD or CAL-and cancers. These findings have important implications for clinicians, especially those providing routine longitudinal care for persons with disability. In particular, these findings support greater vigilance in screening persons with disability for cancers using established screening tests, and investigating new symptoms that arise.

The NHIS data provide no insight into the reasons for these findings. Other research suggests possibilities that merit further exploration. For instance, the later age at diagnosis for persons with disability may relate to delays in cancer diagnosis for this population, as other disadvantaged groups have experienced. ${ }^{21}$ Disparities in provision of screening and preventive services could also contribute to these observations. The US Preventive Services Task Force (USPSTF) strongly recommends screening for CRC in specified populations. ${ }^{28}$ Analysis of 2013 NHIS data suggest that people with mobility disability experience reduced rates of CRC screening, with an inverse relationship between severity of mobility disability and odds of CRC screening. ${ }^{29}$ However, analysis of NHIS data from 1998 through 2010 does not suggest a disparity in rates of CRC screening between people with and without disability. ${ }^{30}$ Screening for the other 3 cancers studied here is not recommended by the USPSTF. Evidence does indicate disability disparities for cervical and breast cancer screening, which are both recommended by USPSTF ${ }^{31,32}$ These observations could raise broader questions about how physicians approach care of patients with disability.

One possible contributor to our findings is diagnostic overshadowing; that is, misattributing new signs and symptoms to a patients' underlying disability rather than a comorbid health condition, and thus delaying diagnosis. Diagnostic overshadowing has been explored in individuals with developmental ${ }^{33-35}$ and mental health disabilities, ${ }^{36-39}$ albeit primarily addressing new or acute problems, not cancer. Among members of the disability community, anecdotal reports suggest that diagnostic overshadowing might also delay cancer diagnoses among persons with physical disability, ${ }^{23}$ but this possibility requires more investigation.

NHIS data unfortunately do not include stage at diagnosis, which would have allowed us to look explicitly at delayed diagnosis. Furthermore, NHIS relies on selfreport, without confirmation of cancer diagnoses. To explore whether NHIS cancer self-reports are credible, we compared our NHIS cancer prevalence rates with those generated from the 2016 SEER cancer registry. ${ }^{40}$ 
Table 3. Sociodemographic Characteristics and Cancer Duration by Cancer Type and Disability Status ${ }^{\text {a }}$

Disability Status

\begin{tabular}{|c|c|c|c|c|c|}
\hline \multirow[b]{2}{*}{ Cancer } & \\
\hline & $\begin{array}{c}\text { No Disability } \\
\% \text { (SE) }\end{array}$ & $\begin{array}{c}\text { MD } \\
\% \text { (SE) }\end{array}$ & $P$ Value ${ }^{b}$ & $\begin{array}{l}\text { CAL } \\
\% \text { (SE) }\end{array}$ & $P$ Value ${ }^{b}$ \\
\hline \multicolumn{6}{|l|}{ Colorectal } \\
\hline Total, N & 515 & 549 & & 410 & \\
\hline Mean age (SE), $y$ & $64.6(0.7)$ & $69.6(0.7)$ & $<.0001$ & $68.5(0.9)$ & $<.0001$ \\
\hline Age & & & $<.0001$ & & .001 \\
\hline$<45 y$ & $7.4(1.6)$ & $3.3(1.3)$ & & $4.4(1.8)$ & \\
\hline $45-64 y$ & $39.0(2.9)$ & $31.5(2.6)$ & & $34.8(3.2)$ & \\
\hline $65-79$ y & $39.3(2.7)$ & $37.7(2.7)$ & & $34.3(2.9)$ & \\
\hline$\geq 80 y$ & $14.3(1.6)$ & $27.5(2.4)$ & & $26.4(2.6)$ & \\
\hline Sex & & & .14 & & .26 \\
\hline Male & $54.2(2.8)$ & $48.4(2.7)$ & & $49.4(3.2)$ & \\
\hline Female & $45.8(2.8)$ & $51.6(2.7)$ & & $50.6(3.2)$ & \\
\hline Race & & & .14 & & .56 \\
\hline White & $83.3(1.9)$ & $84.3(1.9)$ & & $82.8(2.2)$ & \\
\hline Black & $10.3(1.5)$ & $9.4(1.3)$ & & $11.0(1.6)$ & \\
\hline Asian & $4.7(1.1)$ & $2.4(0.9)$ & & $3.2(1.2)$ & \\
\hline Other/Mixed & $1.7(0.8)$ & $4.0(1.2)$ & & $3.1(1.0)$ & \\
\hline Ethnicity & & & .72 & & .79 \\
\hline Hispanic & $5.4(1.1)$ & $6.0(1.2)$ & & $5.9(1.4)$ & \\
\hline Education & & & $<.0001$ & & $<.0001$ \\
\hline Less than high school & $10.0(1.5)$ & $20.6(2.0)$ & & $20.7(2.4)$ & \\
\hline High school & $27.3(2.3)$ & $30.1(2.6)$ & & $31.8(3.0)$ & \\
\hline Some college/associate's degree & $28.6(2.5)$ & $30.4(2.7)$ & & $28.2(3.0)$ & \\
\hline College/Advanced degree & $34.1(2.7)$ & $18.9(2.2)$ & & $19.3(2.7)$ & \\
\hline Body mass index & & & $<.01$ & & .03 \\
\hline$<25 \mathrm{~kg} / \mathrm{m}^{2}$ & $31.9(2.5)$ & $26.8(2.4)$ & & $27.1(2.9)$ & \\
\hline $25-29 \mathrm{~kg} / \mathrm{m}^{2}$ & $40.4(2.9)$ & $32.8(2.8)$ & & $33.2(3.2)$ & \\
\hline $30-34 \mathrm{~kg} / \mathrm{m}^{2}$ & $17.4(2.2)$ & $21.2(2.2)$ & & $18.4(2.5)$ & \\
\hline $35-39 \mathrm{~kg} / \mathrm{m}^{2}$ & $5.1(1.2)$ & $10.0(1.6)$ & & $9.5(1.9)$ & \\
\hline$\geq 40 \mathrm{~kg} / \mathrm{m}^{2}$ & $5.1(2.1)$ & $9.2(1.5)$ & & $11.7(2.1)$ & \\
\hline Smoking status & & & .02 & & .01 \\
\hline Current smoker & $6.9(1.2)$ & $12.4(1.6)$ & & $14.2(2.1)$ & \\
\hline Former smoker & $43.6(2.9)$ & $46.2(2.8)$ & & $44.8(3.3)$ & \\
\hline Never-smoker & $49.6(2.9)$ & $41.4(2.8)$ & & $41.0(3.2)$ & \\
\hline Family income below FPT & $5.1(1.2)$ & $15.4(1.7)$ & $<.0001$ & $18.9(2.2)$ & $<.0001$ \\
\hline Has health insurance & $96.5(0.9)$ & $98.9(0.5)$ & .03 & $98.9(0.6)$ & .04 \\
\hline Has a usual source of care & $97.3(0.7)$ & $98.6(0.5)$ & .11 & $98.3(0.6)$ & .27 \\
\hline Cancer duration, mean (SE), y & $8.6(0.5)$ & $7.0(0.4)$ & $<.01$ & $7.3(0.5)$ & .05 \\
\hline
\end{tabular}


Table 3. Sociodemographic Characteristics and Cancer Duration by Cancer Type and Disability Status (cont.)

\begin{tabular}{|c|c|c|c|c|c|}
\hline \multirow[b]{2}{*}{ Cancer } & \multicolumn{5}{|c|}{ Disability Status } \\
\hline & $\begin{array}{c}\text { No Disability } \\
\% \text { (SE) }\end{array}$ & $\begin{array}{c}\text { MD } \\
\% \text { (SE) }\end{array}$ & $P$ Value $^{b}$ & $\begin{array}{c}\text { CAL } \\
\% \text { (SE) }\end{array}$ & $P$ Value $^{\mathrm{b}}$ \\
\hline \multicolumn{6}{|l|}{ Non-Hodgkin's Lymphoma } \\
\hline Total, N & 308 & 210 & & 148 & \\
\hline Mean age (SE), y & $54.8(1.1)$ & $68.1(1.1)$ & $<.0001$ & $66.7(1.5)$ & $<.0001$ \\
\hline Age category & & & $<.0001$ & & $<.0001$ \\
\hline$<45$ y & $25.5(3.0)$ & $6.9(2.1)$ & & $6.8(2.6)$ & \\
\hline $45-64$ y & $45.0(3.6)$ & $29.0(3.9)$ & & $34.4(4.9)$ & \\
\hline $65-79$ y & $21.5(2.8)$ & $42.1(4.2)$ & & $36.8(4.6)$ & \\
\hline$\geq 80 y$ & $8.1(2.0)$ & $21.9(3.4)$ & & $22.0(4.1)$ & \\
\hline Sex & & & .07 & & .20 \\
\hline Male & $59.6(3.5)$ & $49.6(4.3)$ & & $51.6(5.1)$ & \\
\hline Female & $40.4(3.5)$ & $50.4(4.3)$ & & $48.4(5.1)$ & \\
\hline Race & & & .23 & & .41 \\
\hline White & $88.3(2.4)$ & $87.7(2.8)$ & & $87.5(3.4)$ & \\
\hline Black & $6.4(1.7)$ & $10.1(2.6)$ & & $9.5(3.1)$ & \\
\hline Asian & $4.1(1.6)$ & $1.0(0.8)$ & & $1.4(1.1)$ & \\
\hline Other/Mixed & $1.1(0.6)$ & $1.2(1.0)$ & & $1.7(1.3)$ & \\
\hline Ethnicity & & & .89 & & .77 \\
\hline Hispanic & $8.6(2.6)$ & $9.1(2.6)$ & & $9.8(3.1)$ & \\
\hline Education & & & $<.01$ & & $<.001$ \\
\hline Less than high school & $8.7(2.1)$ & $15.1(3.0)$ & & $17.2(3.8)$ & \\
\hline High school & $23.1(3.3)$ & $31.7(4.0)$ & & $35.2(5.0)$ & \\
\hline Some college/associate's degree & $28.5(3.4)$ & $33.6(4.0)$ & & $31.8(4.7)$ & \\
\hline College/Advanced degree & $39.8(3.9)$ & $19.7(3.5)$ & & $15.9(4.1)$ & \\
\hline Body mass index & & & .85 & & .94 \\
\hline$<25 \mathrm{~kg} / \mathrm{m}^{2}$ & $35.8(3.5)$ & $31.0(4.0)$ & & $34.0(5.1)$ & \\
\hline $25-29 \mathrm{~kg} / \mathrm{m}^{2}$ & $34.2(3.4)$ & $35.2(4.4)$ & & $31.8(4.7)$ & \\
\hline $30-34 \mathrm{~kg} / \mathrm{m}^{2}$ & $21.4(3.1)$ & $23.4(3.7)$ & & $25.1(4.7)$ & \\
\hline $35-39 \mathrm{~kg} / \mathrm{m}^{2}$ & $5.2(1.6)$ & $5.0(1.8)$ & & $4.3(2.1)$ & \\
\hline$\geq 40 \mathrm{~kg} / \mathrm{m}^{2}$ & $3.4(1.1)$ & $5.4(2.4)$ & & $4.9(2.9)$ & \\
\hline Smoking status & & & $<.001$ & & $<.001$ \\
\hline Current smoker & $9.8(2.0)$ & $16.1(3.1)$ & & $22.5(4.5)$ & \\
\hline Former smoker & $26.3(3.1)$ & $39.7(4.0)$ & & $37.6(4.8)$ & \\
\hline Never-smoker & $63.9(3.4)$ & $44.2(4.0)$ & & $39.9(4.9)$ & \\
\hline Family income below FPT & $4.4(1.4)$ & $9.4(2.0)$ & .04 & $13.9(3.5)$ & .01 \\
\hline Has health insurance & $92.8(2.1)$ & $99.2(0.6)$ & $<.01$ & $98.9(0.8)$ & $<.01$ \\
\hline Has a usual source of care & $93.1(1.9)$ & $97.7(1.5)$ & .06 & $98.6(0.9)$ & .01 \\
\hline Cancer duration, mean (SE) & $10.0(0.6)$ & $7.1(0.7)$ & $<.01$ & $7.4(0.8)$ & 0.01 \\
\hline
\end{tabular}




\section{Table 3. Sociodemographic Characteristics and Cancer Duration by Cancer Type and Disability Status ${ }^{\text {a }}$} (cont.)

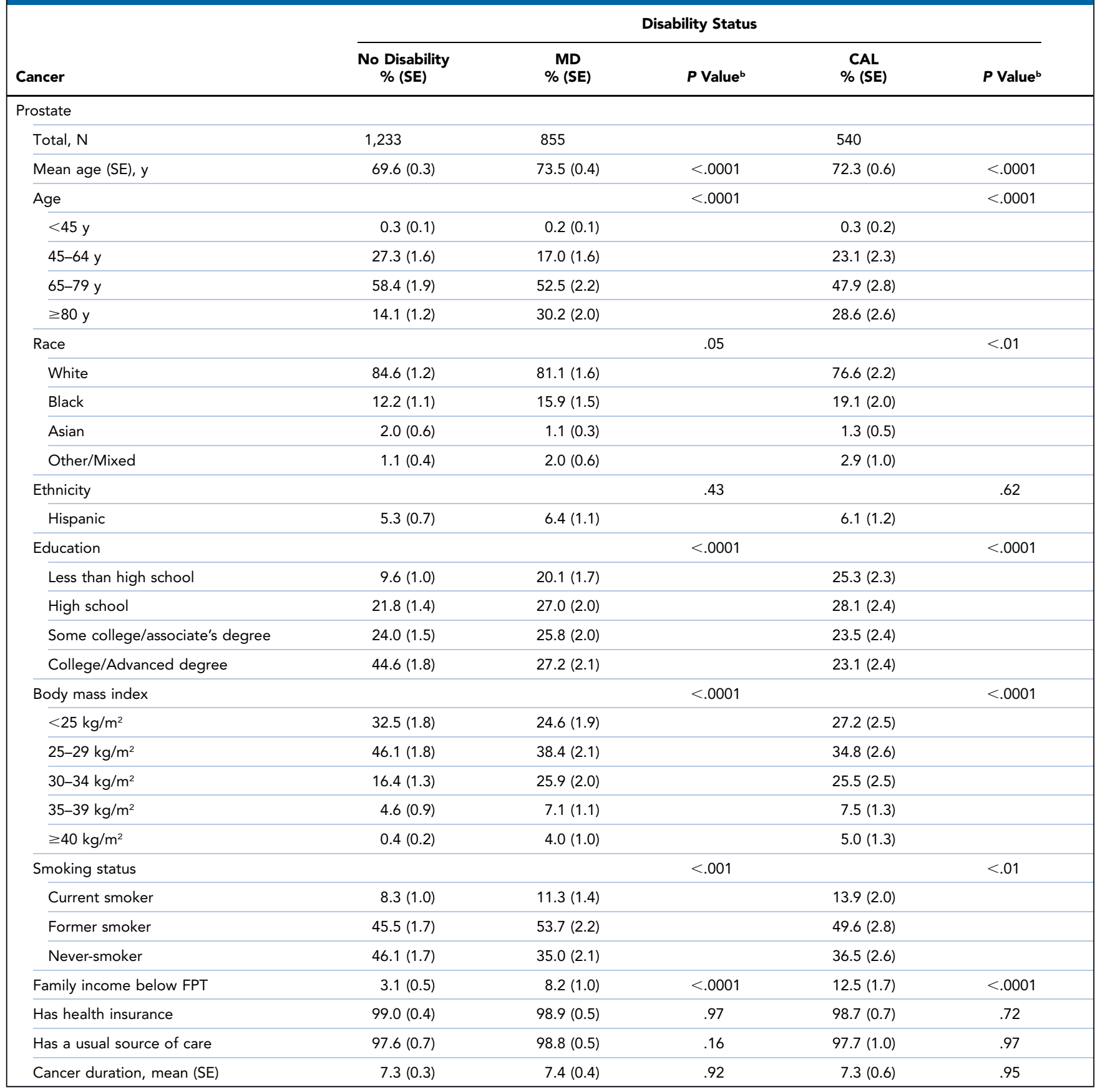

(continued on next page) 


\begin{tabular}{|c|c|c|c|c|c|}
\hline \multirow[b]{2}{*}{ Cancer } & \multicolumn{5}{|c|}{ Disability Status } \\
\hline & $\begin{array}{c}\text { No Disability } \\
\% \text { (SE) }\end{array}$ & $\begin{array}{c}\text { MD } \\
\% \text { (SE) }\end{array}$ & $P$ Value ${ }^{b}$ & $\begin{array}{c}\text { CAL } \\
\% \text { (SE) }\end{array}$ & $P$ Value $^{\text {b }}$ \\
\hline \multicolumn{6}{|l|}{ Ovarian } \\
\hline Total, N & 207 & 149 & & 112 & \\
\hline Mean age (SE), y & $53.3(1.5)$ & $57.4(2.3)$ & $<.0001$ & $59.2(2.3)$ & $<.0001$ \\
\hline Age & & & .14 & & .08 \\
\hline$<45 y$ & $33.6(4.4)$ & $23.3(5.6)$ & & $19.9(5.8)$ & \\
\hline $45-64$ y & $39.0(4.6)$ & $34.1(5.3)$ & & $31.9(6.5)$ & \\
\hline $65-79 y$ & $23.2(4.1)$ & $31.8(5.7)$ & & $34.5(7.2)$ & \\
\hline$\geq 80 y$ & $4.2(1.6)$ & $10.9(3.8)$ & & $13.7(5.2)$ & \\
\hline Race & & & .12 & & .28 \\
\hline White & $79.7(3.9)$ & $89.7(2.7)$ & & $87.3(3.3)$ & \\
\hline Black & $11.1(3.0)$ & $8.3(2.5)$ & & $9.8(3.0)$ & \\
\hline Asian & $4.9(2.5)$ & $0.7(0.5)$ & & $0.7(0.5)$ & \\
\hline Other/Mixed & $4.3(1.8)$ & $1.3(0.7)$ & & $2.2(1.1)$ & \\
\hline Ethnicity & & & .04 & & $<.01$ \\
\hline Hispanic & $14.2(3.6)$ & $5.2(2.4)$ & & $3.2(1.4)$ & \\
\hline Education & & & .18 & & .08 \\
\hline Less than high school & $10.9(3.4)$ & 20.0 & & $26.3(5.7)$ & \\
\hline High school & $26.5(3.6)$ & $31.4(5.4)$ & & $27.0(5.9)$ & \\
\hline Some college/associate's degree & $35.2(4.5)$ & $30.4(5.4)$ & & $27.2(5.3)$ & \\
\hline College/Advanced degree & $27.5(4.2)$ & $18.2(6.1)$ & & $19.4(8.0)$ & \\
\hline Body mass index & & & $<.01$ & & .17 \\
\hline$<25 \mathrm{~kg} / \mathrm{m}^{2}$ & $43.0(5.2)$ & $27.3(5.9)$ & & $32.7(8.1)$ & \\
\hline $25-29 \mathrm{~kg} / \mathrm{m}^{2}$ & $27.9(4.9)$ & $26.5(5.7)$ & & $23.8(5.8)$ & \\
\hline $30-34 \mathrm{~kg} / \mathrm{m}^{2}$ & $21.1(4.2)$ & $19.3(4.3)$ & & $22.1(5.2)$ & \\
\hline $35-39 \mathrm{~kg} / \mathrm{m}^{2}$ & $5.1(1.8)$ & $11.3(3.6)$ & & $9.9(3.3)$ & \\
\hline$\geq 40 \mathrm{~kg} / \mathrm{m}^{2}$ & $2.8(1.3)$ & $15.6(4.1)$ & & $11.5(4.2)$ & \\
\hline Smoking status & & & .20 & & .15 \\
\hline Current smoker & $21.6(3.6)$ & $20.5(3.9)$ & & $25.4(5.6)$ & \\
\hline Former smoker & $18.9(3.6)$ & $30.9(5.8)$ & & $31.2(7.4)$ & \\
\hline Never-smoker & $59.4(4.5)$ & $48.6(6.2)$ & & $43.4(7.0)$ & \\
\hline Family income below FPT & $7.7(2.4)$ & $30.6(5.1)$ & $<.0001$ & $32.7(6.0)$ & $<.0001$ \\
\hline Has health insurance & $88.4(3.2)$ & $94.2(2.2)$ & .13 & $93.3(3.3)$ & .30 \\
\hline Has a usual source of care & $92.1(2.7)$ & $97.6(1.3)$ & .06 & $95.9(2.9)$ & .33 \\
\hline Cancer duration, mean (SE) & $13.1(1.3)$ & $11.0(1.5)$ & .29 & $12.2(1.8)$ & .69 \\
\hline
\end{tabular}

Abbreviations: CAL, complex activity limitation; FPT, federal poverty threshold; MD, movement difficulty; NHIS, National Health Interview Survey. aAll analyses accounted for the complex sampling design and used NHIS sampling weights to produce nationally representative estimates for civilian, noninstitutionalized US residents.

${ }^{\mathrm{b}}$ Comparing persons with no disability versus those with MD or CAL disability.

We found similar CRC and prostate cancer prevalence estimates across the 2 data sources, whereas the NHIS estimates higher rates for NHL and ovarian cancer (although the absolute differences are small). These findings suggest that NHIS participants are not underreporting cancer diagnoses, although-especially for ovarian cancer-overreporting could occur.

In addition to concerns about the accuracy of cancer reports, our findings share the limitations of other crosssectional survey data analyses. We identify statistical 


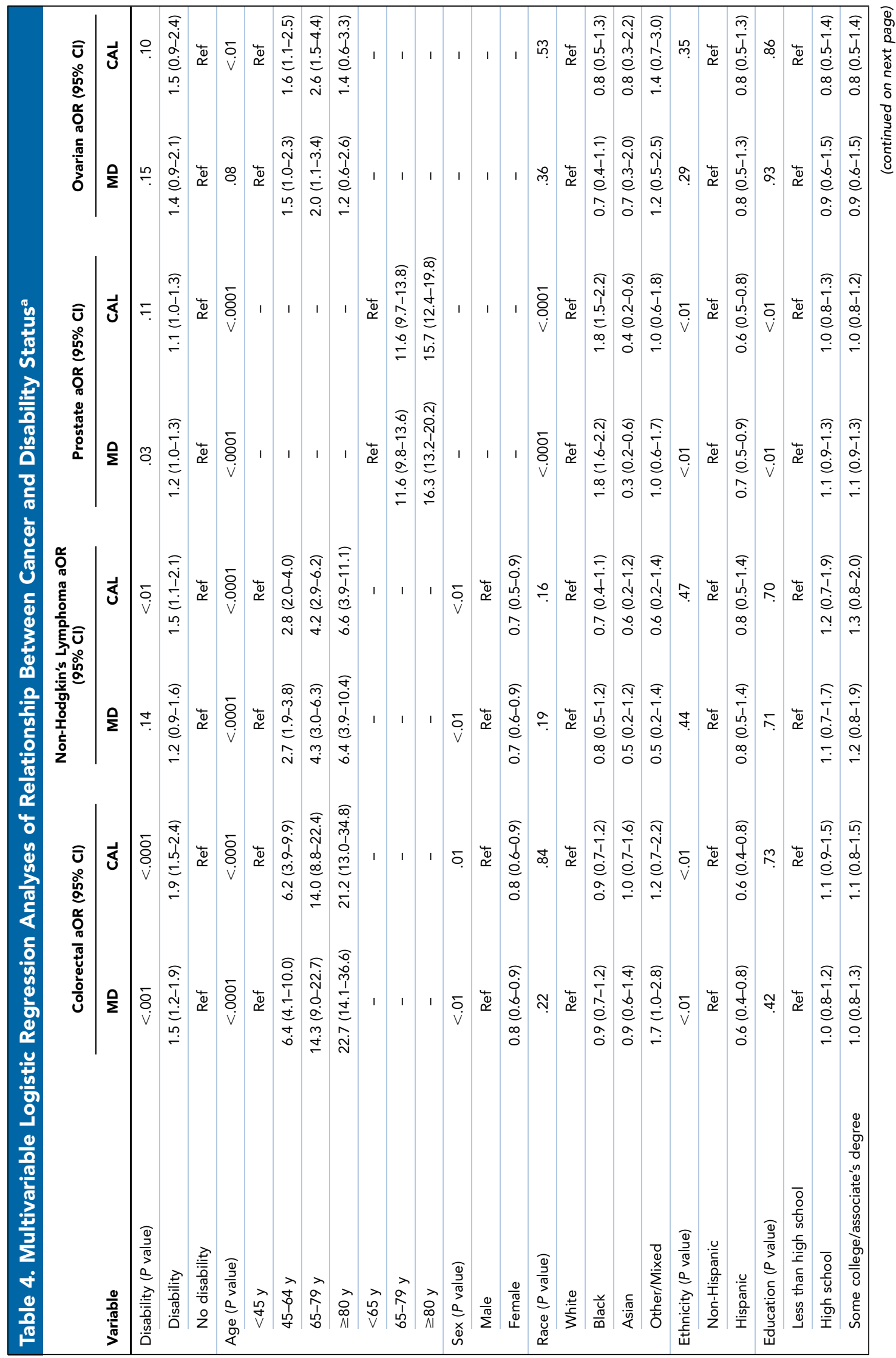




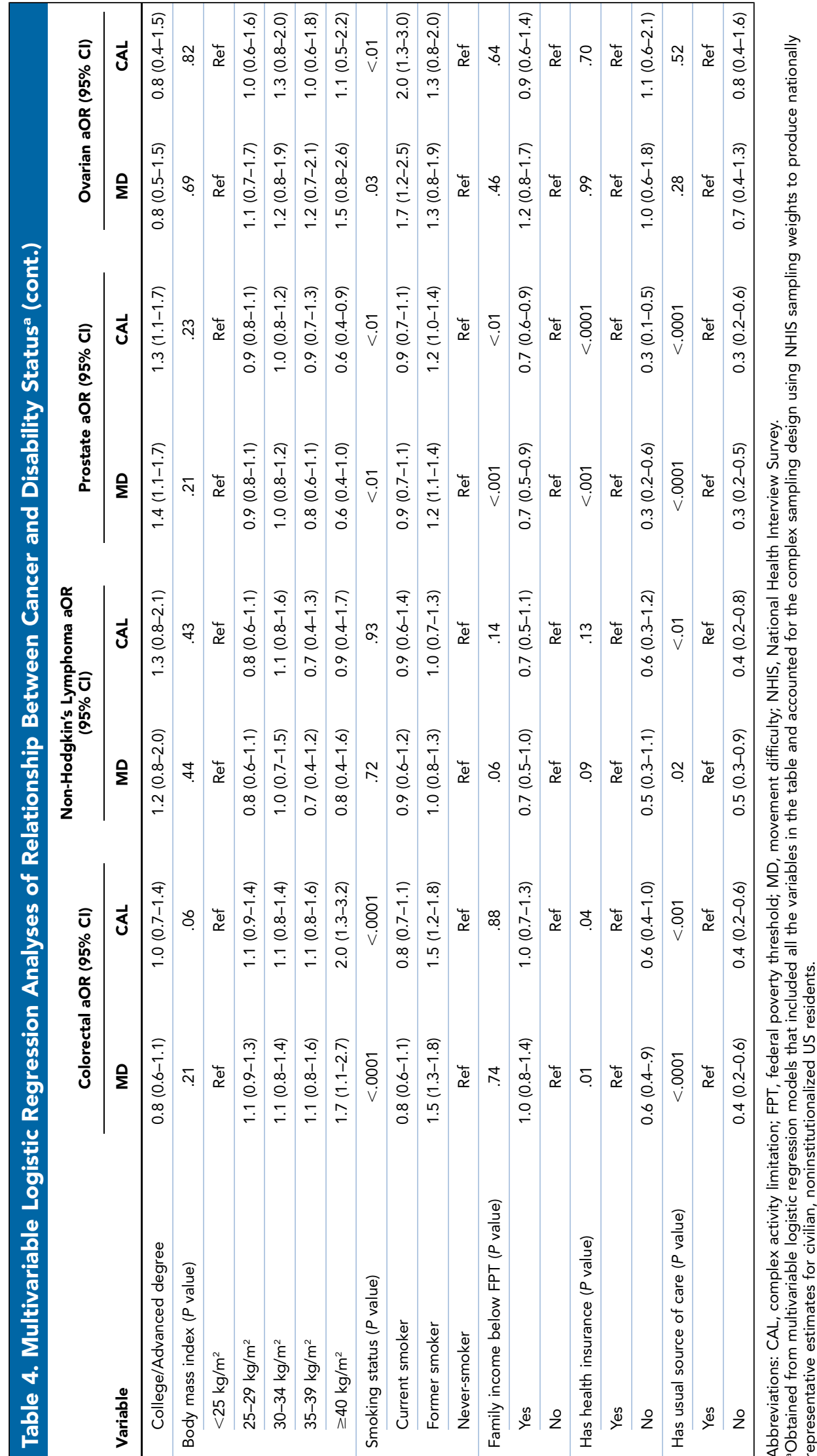


associations, not causality. We cannot precisely assess whether disability occurred because of cancer or before the cancer, although by looking at different time frames we attempted to explore this timing. Given concerns about potential stigmatization of disability, response bias or sociocultural factors could affect self-reports of functional status. Although we examined 8 years of NHIS data, too few cases reported sensory, mental health, or cognitive disability for us to perform rigorous analyses for these disability groups. Nonetheless, because NHIS data are nationally representative, they allow us to produce population estimates; their detail about cancer diagnoses and functional status also offers the opportunity to explore associations between disability and cancer experiences, which have not been previously assessed.

\section{Conclusions}

Little research has explored cancer among persons with preexisting disability, and our findings raise further questions. Our population-based analyses suggest that persons with disability should perhaps be considered a high-risk population, with higher cancer incidence. Optimizing appropriate screening and fully investigating new signs and symptoms are therefore critical. Given older ages at diagnosis among persons with disability, further investigation into the possibility of diagnostic overshadowing is important. Erroneously attributing new signs and symptoms to a patient's underlying disability could delay cancer diagnoses and reduce the likelihood of a good outcome.

Submitted November 8, 2019; accepted for publication February 18, 2020.

Author contributions: Study concept and design: lezzoni, El-Jawahri. Principal Investigator: lezzoni. Literature reviews, data compilation, database management, and analytic programming: Rao, Agaronnik. Statistical analysis: Rao. Analysis and interpretation of results: lezzoni, Rao, El-Jawahri. Manuscript preparation: lezzoni, Rao. Critical review: Agaronnik, El-Jawahri.

Disclosures: The authors have disclosed that they have not received any financial consideration from any person or organization to support the preparation, analysis, results, or discussion of this article.

Funding: The Eunice Kennedy Shriver National Institute of Child Health and Human Development (R21 HD095240-01) funded this work (L.I.I., Principal Investigator; A.E.J., Co-Investigator).

Disclaimer: The funders were not involved in the design, collection, analysis, interpretation of data, or decision to approve publication of finished manuscript.

Correspondence: Lisa I. lezzoni, MD, MSc, Health Policy Research CenterMongan Institute, Massachusetts General Hospital, 100 Cambridge Street, Suite 1600, Boston, MA 02114. Email: liezzoni@mgh.harvard.edu

\section{References}

1. Centers for Disease Control and Prevention. Disability impacts all of us. Accessed October 18, 2019. Available at: https://www.cdc.gov/ncbddd/ disabilityandhealth/infographic-disability-impacts-all.html

2. National Center for Health Statistics. National Health Interview Survey, 2017. Public-use data file and documentation. Accessed July 15, 2020. Available at: https://www.cdc.gov/nchs/nhis/data-questionnaires-documentation.htm

3. Heron M. Deaths: leading causes for 2016. Natl Vital Stat Rep 2018;67:1-77

4. Stout NL, Silver JK, Alfano CM, et al. Long-term survivorship care after cancer treatment: a new emphasis on the role of rehabilitation services. Phys Ther 2019;99:10-13.

5. Sharma R, Molinares-Mejia D, Khanna A, et al. Training and practice patterns in cancer rehabilitation: a survey of physiatrists specializing in oncology care. PM R 2020;12:180-185

6. Marshall TF, Alfano CM, Sleight AG, et al. Consensus-building efforts to identify best tools for screening and assessment for supportive services in oncology. Disabil Rehabil 2019;(Fe 19):1-8.

7. Social Security Administration. All disabled beneficiaries-distribution, by sex and diagnostic group, December 2017. In: Annual Statistical Report on the Social Security Disability Insurance Program; 2018:25. Accessed July 17, 2020. Available at: https://www.ssa.gov/policy/docs/statcomps/ di_asr/2017/di_asr17.pdf.

8. Social Security Administration. Compassionate allowances. Accessed October 18, 2019. Available at: https://www.ssa.gov/ compassionateallowances/

9. Centers for Disease Control and Prevention. Cigarette smoking among adults with disabilities. Accessed October 18, 2019. Available at: https:// www.cdc.gov/ncbddd/disabilityandhealth/smoking-in-adults.html

10. Centers for Disease Control and Prevention. Overweight and obesity-among people with disabilities. Accessed October 18, 2019 Available at: https://www.cdc.gov/ncbddd/disabilityandhealth/ documents/obesityFactsheet2010.pdf

11. National Cancer Institute. Tobacco. Accessed October 18, 2019. Available at: https://www.cancer.gov/about-cancer/causes-prevention/risk/tobacco

12. National Cancer Institute. Obesity. Accessed October 18, 2019. Available at: https://www.cancer.gov/about-cancer/causes-prevention/risk/obesity

13. National Council on Disability. Highlighting disability/ poverty connection, NCD urges Congress to alter federal policies that disadvantage people with disabilities. Accessed October 18, 2019. Available at: https:// ncd.gov/newsroom/2017/disability-poverty-connection-2017-progressreport-release

14. Krieger N. Defining and investigating social disparities in cancer: critical issues. Cancer Causes Control 2005;16:5-14

15. Steele CB, Townsend JS, Courtney-Long EA, et al. Prevalence of cancer screening among adults with disabilities, United States, 2013. Prev Chronic Dis 2017;14:E09.

16. Andresen EM, Peterson-Besse JJ, Krahn GL, et al. Pap, mammography, and clinical breast examination screening among women with disabilities: a systematic review. Womens Health Issues 2013;23:e205-214.

17. lezzoni LI, Kurtz SG, Rao SR. Trends in Pap testing over time for women with and without chronic disability. Am J Prev Med 2016;50:210-219.

18. Institute of Medicine Committee (IOM), The Future of Disability in America. Washington, DC: National Academies Press; 2007.

19. Molton IR, Jensen MP. Aging and disability: biopsychosocial perspectives. Phys Med Rehabil Clin N Am 2010;21:253-265.

20. LaPlante MP. Key goals and indicators for successful aging of adults with early-onset disability. Disabil Health J 2014;7(1 Suppl)S44-50.

21. Bradley CJ, Given CW, Roberts C. Disparities in cancer diagnosis and survival. Cancer 2001;91:178-188

22. Mullins MA, Peres LC, Alberg AJ, et al. Perceived discrimination, trust in physicians, and prolonged symptom duration before ovarian cancer diagnosis in the African American Cancer Epidemiology Study. Cancer 2019;125:4442-4451.

23. lezzoni LI. Dangers of diagnostic overshadowing. N Engl J Med 2019;380: 2092-2093

24. National Center for Health Statistics. Survey Description, National Health Interview Survey, 2017. Hyattsville, MD: Centers for Disease Control and Prevention; 2018.

25. Kraus L, Lauer E, Coleman R, et al. 2017 Disability Statistics Annual Report. Durham, NH: University of New Hampshire; 2018.

26. National Cancer Institute. Age and cancer risk. Accessed October 18, 2019. Available at: https://www.cancer.gov/about-cancer/ causes-prevention/risk/age

27. Fleisch Marcus A, Illescas AH, Hohl BC, et al. Relationships between socia isolation, neighborhood poverty, and cancer mortality in a populationbased study of US adults. PLoS One 2017;12:e0173370. 
28. Bibbins-Domingo K, Grossman DC, Curry SJ, et al. Screening for colorectal cancer: US Preventive Services Task Force recommendation statement. JAMA 2016;315:2564-2575.

29. Gofine M, Mielenz TJ, Vasan S, et al. Use of colorectal cancer screening among people with mobility disability. J Clin Gastroenterol 2018;52: 789-795.

30. lezzoni LI, Kurtz SG, Rao SR. Trends in colorectal cancer screening over time for persons with and without chronic disability. Disabil Health J 2016 ; 9:498-509.

31. Horner-Johnson W, Dobbertin K, lezzoni LI. Disparities in receipt of breast and cervical cancer screening for rural women age 18 to 64 with disabilities. Womens Health Issues 2015;25:246-253.

32. Horner-Johnson W, Dobbertin K, Andresen EM, et al. Breast and cervical cancer screening disparities associated with disability severity. Womens Health Issues 2014;24:e147-153.

33. Jones S, Howard L, Thornicroft G. 'Diagnostic overshadowing': worse physical health care for people with mental illness. Acta Psychiatr Scand 2008;118:169-171.

34. Foley K-R, Trollor J. Management of mental ill health in people with autism spectrum disorder. Aust Fam Physician 2015;44:784-790.
35. Kerns CM, Kendall PC, Zickgraf H, et al. Not to be overshadowed or overlooked: functional impairments associated with comorbid anxiety disorders in youth with ASD. Behav Ther 2015;46:29-39.

36. Happell B, Ewart SB, Bocking J, et al. 'That red flag on your file': misinterpreting physical symptoms as mental illness. J Clin Nurs 2016;25 2933-2942.

37. Shefer G, Henderson C, Howard LM, et al. Diagnostic overshadowing and other challenges involved in the diagnostic process of patients with mental illness who present in emergency departments with physical symptoms—a qualitative study. PLoS One 2014;9:e111682.

38. Shefer G, Cross S, Howard LM, et al. Improving the diagnosis of physical illness in patients with mental illness who present in emergency departments: consensus study. J Psychosom Res 2015;78:346-351.

39. van Nieuwenhuizen A, Henderson C, Kassam A, et al. Emergency department staff views and experiences on diagnostic overshadowing related to people with mental illness. Epidemiol Psychiatr Sci 2013;22: 255-262.

40. SEER Explorer: an interactive website for SEER cancer statistics. Surveillance Research Program, National Cancer Institute. Accessed October 18, 2019. Available from https://seer.cancer.gov/explorer/ 
Supplemental online content for:

\section{Cross-Sectional Analysis of the Associations Between Four Common Cancers and Disability}

Lisa I. lezzoni, MD, MSc; Sowmya R. Rao, PhD; Nicole D. Agaronnik, BS; and Areej El-Jawahri, MD

J Natl Compr Canc Netw 2020;18(8):1031-1044

eAppendix 1: Question Stems and Response Categories for the Adult Health Status and Limitations Module eAppendix 2: Definitions of Disability Measures Using National Health Interview Survey Responses 


\section{eAppendix 1. Question Stems and Response Categories for the Adult Health Status and Limitations Module in the Sample Adult Core Questionnaire of the National Health Interview Survey}

"The next questions ask about difficulties you may have doing certain activities because of a HEALTH PROBLEM. By 'health problem' we mean any physical, mental, or emotional problem or illness (not including pregnancy). By yourself, and without using any special equipment, how difficult is it for you to..."

Response categories are: "not at all difficult," “only a little difficult," "somewhat difficult," "very difficult," "can't do at all," "do not do this activity" ("refused" and "don't know").

\section{Categories of disability created by combining responses from functional and activity limitation questions: \\ Basic Action Difficulties (BADs)}

- Movement difficulty (MD): at least somewhat difficult to walk, stand, climb stairs, sit, stoop, reach, grasp, or carry

- Sensory difficulty: trouble seeing even when wearing glasses/contact lenses; or deaf or trouble hearing without a hearing aid

- Emotional difficulty: sad, nervous, restless, hopeless, "everything was an effort," and worthless feelings in the past 30 days

- Cognitive difficulty: limited in any way because of difficulty remembering or because of periods of confusion

\section{Complex Activity Limitations (CALs)}

- Self-care limitation: difficulty with any component of activities of daily living (ADLs) or instrumental ADLs (IADLs)

- Social limitation: going out, participating in social activities or relaxing at least somewhat difficult

- Work limitation: cannot work or has work limitations because of physical, mental or emotional problem 


\section{eAppendix 2. Definitions of Disability Measures Using National Health Interview Survey Responses}

\section{Basic Action Difficulties}

\begin{tabular}{|c|c|}
\hline Movement Disability & $\begin{array}{l}\text { A response of "only somewhat difficult," "very difficult," or "can't do at all" on any of the following questions: } \\
\text { "By yourself, and without using any special equipment, how difficult is it for you to } \\
\ldots \text {. Walk a quarter of a mile-about } 3 \text { city blocks? } \\
\ldots \text {. Walk up } 10 \text { steps without resting? } \\
\text {.. Stand or be on your feet for about } 2 \text { hours? } \\
\text {.. Sit for about } 2 \text { hours? } \\
\text {. . Stoop, bend, or kneel? } \\
\text {. Reach up over your head? } \\
\text {. . Use your fingers to grasp or handle small objects? }\end{array}$ \\
\hline Sensory Disability & $\begin{array}{l}\text { A "yes" response to either of the following questions: } \\
\text { "Do you have any trouble seeing, even when wearing glasses or contact lenses?" If the response is yes: "Are } \\
\text { you blind or unable to see at all?" "Have you ever worn a hearing aid?" } \\
\text { OR a response of "a lot of trouble" or "deaf" to the following: } \\
\text { "Which statement best describes your hearing without a hearing aid: Good, a little trouble, a lot of trouble, deaf?" }\end{array}$ \\
\hline \multirow[t]{2}{*}{ Emotional Disability } & $\begin{array}{l}\text { A score of } 13 \text { or more based on the following questions which comprise the K6 serious psychological distress scale: } \\
\text { "During the past } 30 \text { days, how often did you feel } \\
\ldots \text {. So sad that nothing could cheer you up? } \\
\ldots \text {. Nervous? } \\
\text {.. Restless or fidgety? } \\
\ldots \text {. Hopeless? } \\
\ldots \text {. That everything was an effort? } \\
\text {... Worthless? } \\
\text { Possible answers are } \\
\text { "all of the time" (4 points) } \\
\text { "most of the time" ( } 3 \text { points) } \\
\text { "some of the time" (2 points) } \\
\text { "a little of the time" (1 point) } \\
\text { "none of the time" ( } 0 \text { points). }\end{array}$ \\
\hline & To score the K6, the points are added together yielding a possible total of 0 to 24 points \\
\hline Cognitive Disability & $\begin{array}{l}\text { A "yes" response to the following: } \\
\text { \{Are/Is\} \{person\} LIMITED IN ANY WAY because of difficulty remembering or because }\{\text { you/they }\} \text { e experience periods } \\
\text { of confusion?" }\end{array}$ \\
\hline
\end{tabular}

\section{Complex Activity Limitations}

Self Care Limitation

Require help with any daily living activities:

bathing or showering

dressing

getting in or out of bed or a chair using the toilet

eating and getting around inside the home

OR

Require help with any instrumental daily living activities:

household chores doing necessary business shopping getting around for other purposes

Social Limitation

A response of "only somewhat difficult," "very difficult," or "can't do at all" on any of the following questions:

"By yourself and without using any special equipment, how difficult is it for you to

... Go out to things like shopping, movies, or sporting events?

... Participate in social activities such as visiting friends, attending clubs and meetings, going to parties?

. Do things to relax at home or for leisure (reading, watching TV, sewing, listening to music)?"

Work Limitation

A "yes" response to either of the following:

"Does a physical, mental, or emotional problem NOW keep \{you/anyone in the family (age $\geq 18$ years)\} from working at a job or business?"

" $\{$ Are you/any of these family members\} limited in the kind OR amount of work

\{you/they\} can do because of a physical, mental or emotional problem?" 